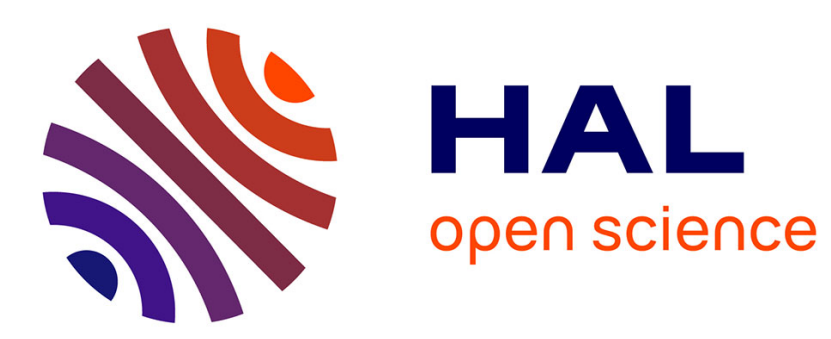

\title{
A Transition from a Microscopic to a Macroscopic Approach to Steady State Detonation C. Coffey
}

\section{To cite this version:}

C. Coffey. A Transition from a Microscopic to a Macroscopic Approach to Steady State Detonation. Journal de Physique IV Proceedings, 1995, 05 (C4), pp.C4-477-C4-483. 10.1051/jp4:1995438 . jpa00253743

\section{HAL Id: jpa-00253743 https://hal.science/jpa-00253743}

Submitted on 1 Jan 1995

HAL is a multi-disciplinary open access archive for the deposit and dissemination of scientific research documents, whether they are published or not. The documents may come from teaching and research institutions in France or abroad, or from public or private research centers.
L'archive ouverte pluridisciplinaire HAL, est destinée au dépôt et à la diffusion de documents scientifiques de niveau recherche, publiés ou non, émanant des établissements d'enseignement et de recherche français ou étrangers, des laboratoires publics ou privés. 


\title{
A Transition from a Microscopic to a Macroscopic Approach to Steady State Detonation
}

\author{
C.S. Coffey \\ Naval Surface Warfare Center, White Oak Laboratory, Silver Spring, MD 20903-5640, U.S.A.
}

\begin{abstract}
Recent efforts to understand initiation of reaction in crystalline explosives on the microscopic level during shock or impact have been extended to the macroscopic case. This allows a different approach to the detonation problem. The initiation portion of the detonation wave is determined and when combined with the reaction zone length completely specifies the detonation wave. Detonation in an unconfined cylinderical charge is treated. This approach should have general application beyond the narrow confines of detonation in predicting the initiation response of crystalline explosives to any arbitrary shock or impact.
\end{abstract}

\section{INTRODUCTION}

The classical analysis of a detonation wave propagating in an explosive charge is well known and gives reasonable predictions of both detonation pressure and detonation velocity [1-4]. However, this analysis is highly idealized. The detonation wave is treated as an infinitely thin discontinuity separating the undisturbed material ahead of the wave from the fully reacted material to its rear. Numerous efforts have since been made to account for reaction zone thickness, reaction rate processes, material sensitivity to shock, detonation wave curvature and many of the other attributes of detonation waves [5-8].

Universally, all of these efforts have focussed on pressure as the essential component driving the initiation process. While pressure is important, recent efforts have identified shear as the mechanism by which energy is dissipated and initiation occurs in the detonation wave front. This suggests a different approach to the detonation problem. The shear driven initiation portion of the detonation wave front is first determined. This is then combined with the reaction zone thickness to describe the complete detonation wave. Here we present a determination of the shear wave and initiation front in an unconfined explosive charge. The reaction zone thickness, which can vary across the reaction front, is described in part by the microscopic analysis and in part by the boundary conditions. The results predict that the reaction zone can readily be measured for real charges. In this picture the high pressure portion of the detonation wave is just the end state of the process and supplies some energy to sustain the shear driven initiation process.

This approach resolves a major inconsistency between current detonation theory and experiment, namely 
that of dead pressing and similar situations in which it is impossible to initiate crystalline explosives at any pressure when plastic flow is prevented and yet the same material detonates easily at pressures of a few tenths of a GPa when shear and plastic flow occurs. Determining initiation due to the energy dissipated during plastic deformation has the potential of solving a broad range of initiation problems arising from an arbitrary impact or shock stimuli.

\subsection{BRIEF REVIEW OF INITIATION AT THE MICROSCOPIC LEVEL}

In an effort to understand initiation, we have identified the localized energy dissipation that occurs within the explosive crystals during plastic deformation due to shock or impact as the mechanism responsible for initiation of reaction [9-11]. This is a shear driven process in which the energy dissipated by the moving dislocations responsible for plastic deformation is localized into small regions of sufficient energy density to start chemical reactions. Specifically, during detonation the local lattice distortions produced by the rapidly moving dislocations driven by the shock of the detonation are able to resonantly excite the internal vibrational modes of the explosive molecules. Rapid multiphonon excitation occurs when the energy of the optical phonons generated by the moving dislocations equals the energy required to excite transitions between the internal molecular vibrational levels, typically $\omega$ $\approx 10^{13} \mathrm{rad} / \mathrm{s}$. The optical phonons generated by the moving dislocations are centered in a band about $\omega$ $=2 \pi \mathrm{v} / \mathrm{d}$. The quantity $\mathrm{d}$ is the molecular spacing, $\mathrm{d} \approx 10^{-9} \mathrm{~m}$, and $\mathrm{v}$ is the dislocation velocity. Here $\mathrm{v}$ $=\mathrm{v}_{0} \exp \left(-\tau_{0} / \tau\right) \cdot{ }^{12,13} \mathrm{v}_{0}$ is the shear wave speed, $\approx 2 \times 10^{3} \mathrm{~m} / \mathrm{s}, \tau$ is the applied shear stress and $\tau_{0}$ is a characteristic shear stress of the material, $\tau_{0} \approx 10 \mathrm{MPa}$ to about $1.0 \mathrm{GPa}$. For a high amplitude shock it can easily occur that $\tau \gg \tau_{0}$ and $\omega \approx 2 \pi v_{0} / \mathrm{d} \approx 10^{13} \mathrm{rad} / \mathrm{s}$. Thus the optical phonons created in this band can resonantly excite the internal molecular modes and cause the rapid molecular dissociation associated with detonations.

Mild impact, $\tau \leq \tau_{0}$, generates only low velocity dislocations and lower plastic strain rates. These lower velocity dislocations can also produce energy localization and ignition but at a much slower rate and are not of treated here.

\section{INITIATION AT THE MACROSCOPIC LEVEL}

Since shear stress is the driver of these microscopic processes, it is possible to use the shear stress to extend these concepts to the macroscopic level and obtain predictions of the pressure wave and the shear stress - initiation profile of a steady state detonation wave as well as predictions of other features including the failure diameter.

Consider the case of a steady state detonation wave propagating with a velocity $\mathrm{D}$ along the axis of an unconfined cylindrical explosive charge of radius $r_{0}$. Let the charge be composed of an aggregate of randomly oriented and sized explosive crystals held in a soft polymer binder. It will be assumed that plastic deformation can always occur since the stresses encountered in a detonation are usually far in excess of the yield strength of any material.

A shear stress can be generated locally within the charge by the contact forces arising from particles pushing against each other due to the shock of the detonation wave [14]. Alternatively, shear stress can arise from the rarefactions originating at the unconfined walls of the charge. For the purposes of this paper it will be assumed that the charge is at or near its theoretical maximum density so that the local shear stresses in the aggregate due to contact forces can be neglected. Here the focus will be on the shear due to the pressure gradient produced by the relief waves that enter the explosive from the unconfined side walls.

Rather than writing the shear stress as the deviatoric elements of a stress tensor, it is more informative to directly write the shear stress in terms of the differential of the forces acting on a crystal element. The shear stress $\vec{\tau}$ can be expressed in terms of the spatial derivative of the force $\vec{F}$ as 


$$
\vec{\tau}=\frac{1}{A}(\overrightarrow{d r} \cdot \vec{\nabla}) \vec{F}
$$

where $A$ is the cross sectional area. The differential element $\overrightarrow{d r}$ is the size of the active plastic deformation region of the crystal. It is this force gradient applied to a crystal that causes the creation and motion of dislocations and plastic flow to occur. Because of the magnitude of the shear stresses and the plastic flow, the components of the force are strongly coupled to each other which allows the simplification

$$
\vec{\tau} \approx(\vec{d} r \cdot \vec{\nabla}) \vec{P}
$$

where $\vec{P}=\vec{P}(r, t)$ is the pressure. If the pressure is written as $\vec{P}=P \hat{u}$ where $\hat{u}$ is any unit vector and $P$ is the pressure amplitude, then

$$
\vec{\tau}=\hat{u}(\overrightarrow{d r} \cdot \vec{\nabla}) P
$$

and the amplitude of the shear stress becomes

$$
\tau=\langle\overrightarrow{d r} \cdot \vec{\nabla}\rangle P
$$

In order for a steady state detonation to develop, initiation must proceed at a constant rate on the surface of the initiation portion of the detonation wave. To achieve this constant rate of molecular excitation and dissociation requires that the shear stress be a constant over the entire surface of the initiation front. Thus on the surface of the initiation portion of a steady state detonation wave $d \tau=0$ or

$$
d \tau=\overrightarrow{d r} \cdot \vec{\nabla} \tau=0
$$

Combining equations (4) and (5) gives the condition for a steady state detonation wave as

$$
\nabla^{2} P=0
$$

For a cylinderical charge this has the solution

$$
P(r, z)=P_{0} J_{0}(\alpha r) e^{\alpha z}
$$

where $\mathrm{J}_{0}(\alpha r)$ is the zero order Bessel function and $\mathrm{P}_{0}$ is the pressure amplitude of the steady detonation 
wave. This closely resembles the form of recent empirical fits to the experimental data for the pressure field of a steady state detonation in a cylindrical charge $[15,16]$.

The magnitude of the shear stress is

$$
|\tau|=\alpha P_{0} e^{\alpha z}\left(J_{1}(\alpha r)^{2}+J_{0}(\alpha r)^{2}\right)^{1 / 2} d r
$$

For an aggregate of randomly oriented explosives crystals it is only necessary that the magnitude of the shear stress be a constant on the initiation surface ahead of the steady state detonation wave. Thus, on the initiation front the magnitude of the shear stress on the axis of the charge, $r=0, z=0$, and at any other point $(r, z)$ on the constant shear/initiation surface are equal

$$
|\tau(r=0, z=0)|=|\tau(r, z)|
$$

which reduces to

$$
e^{-\alpha z}=\left(J_{1}(\alpha I)^{2}+J_{0}(\alpha I)^{2}\right)^{1 / 2}
$$

It has tactitly been assumed that the shear stress on the surface of interest is sufficient to cause initiation, $\tau \geq \tau_{c}$, where $\tau_{c}$ is the critical shear stress necessary for initiation.

\section{STEADY STATE DETONATION}

In order to sustain a steady state detonation the boundary conditions imposed by an unconfined charge require that detonation must exist at the edge of the cylinder. This is necessary in order that the detonation reaction supply energy to the wave front to offset the energy lost to the rarefactions at the edge of the charge. Often in a real charge there appears to be a thin shell of explosive material on the outer edge of the charge that does not undergo reaction [17]. This is probably due to the length of the reaction zone and will not be dealt with further here.

Typically, the charge is driven to detonation by a plane shock wave. Assuming this to be the case, initiation of chemical reaction cannot first occur at the shock front in the interior of the charge since initially the gradient of the pressure is zero in this region and consequently so is the shear stress. Rather, initiation must first occur at the edge of the charge where the rarefaction waves entering from the unconfined side walls create a pressure gradient and consequently a shear stress and plastic flow. A steady state detonation develops as the rarefaction waves progress towards the center of the charge and back to the edge to eventually establish the shear stress profile given by equations (8) or (10).

\subsection{REACTION ZONE LENGTH}

The reaction zone length, $z_{0}$, is determined mainly by the microscopic processes of initiation [9-11,14]. This length is measured from the initiating shear wave front given in equation (8) and extends to the point where the reaction is completed which is usually at the detonation front. Because of the boundary conditions $z_{0}$ varies from the center to the edge of the unconfined charge, $z_{0}=z_{0}(r)$. For simplicity, the reaction zone length along the axis of the charge will be taken to be independent of the boundary conditions and is the characteristic reaction length, $\delta$, of the explosive, $z_{0}(0)=\delta$. At the edge of the charge the reaction zone thickness is controlled in part by the boundary conditions which moderate the 
shear stress that drives the reaction.

The shear/initiation front is determined by the shear due to the pressure gradient and is supported by the energy released in the reaction zone. To maximize the shear and associated reaction rate and minimize the energy flow between the interior and the edge of the charge, it is proposed that in the steady state the reaction at a position on the axis of the charge, $(0, z)$, is just completed as the reactions at positions on the edge of the charge with the same $z$ coordinate, $\left(r_{0}, z\right)$, are just initiated. Thus, in a coordinate system centered at the initiation front on the axis of the charge and moving with that front, the shear stress at the edge of the charge is determined from equation (8) with $z=-\delta$. Initiation occurs when a critical shear stress amplitude is achieved, $\tau \geq \boldsymbol{\tau}_{\mathrm{c}}$. The plastic deformation and associated energy dissipation that occur prior to initiation are the same as would occur in a similar inert material and determine the energy required to ignite the sample.

Since shear arising from the rarefactions entering from the edge of the unconfined charge is required to establish a steady state detonation, the reaction at the edge of the charge develops for twice the time interval as the reaction in the center. Thus at the edge of the charge the reaction zone thickness is twice that at the center, $z_{0}\left(r_{0}\right)=2 \delta$. If it is assumed that the reaction goes to completion at the detonation front then the distance that the steady state detonation front at the edge of the charge lags the detonation front at the center of the charge is $2 \delta$. In a coordinate system centered on the axis of the charge at the detonation front and moving with that front, the pressure at the edge of the charge is determined from equation (7) with $\mathrm{z}=-2 \delta$.

Equations (7), (8) and (10) along with the reaction zone length, $\delta$, define the surfaces of steady state detonation and initiation waves propagating along the axis of an unconfined cylindrical charge. Table (1) show some comparisons between experimental data obtained from two different explosives, Comp $\mathrm{B}$ and an ammonium perchlorate and aluminum, $\mathrm{AP} / \mathrm{Al}$, based explosive and predictions obtained from the above equations. This indicates that within the probable accuracy of the data the characteristic reaction zone length, $\delta$, is nearly a constant independent of charge diameter for the AP/Al based explosive. Also, within the accuracy of the data, the detonation front at the edge of the charge lags the detonation front at the center of the charge by the predicted amount $2 \delta$ for both explosives.

Finally, consider briefly the case when the cylindrical charge is enclosed in a thick walled tube so that the rarefaction waves from the side walls are reduced. With sufficient confinement the pressure gradient in the charge vanishes and along with it the shear stress within the charge. In the absence of shear and plastic deformation, energy localization and the initiation of chemical reaction cannot occur regardless of the amplitude of the shock introduced into the charge. There exists a substantial amount of experimental data from shock experiments in heavily confined charges at near theoretical maximum density that support this prediction [18-21].

\section{SUMMARY}

The observation that plastic deformation and the associated shear stress are responsible for energy dissipation and localization and the subsequent initiation of explosive crystals has been carried to the macroscopic level to describe the initiation of a steady state detonation wave in an unconfined cylinderical charge. Together the initiation front and the reaction zone length completely specify the detonation wave. Predictions have been obtained that agree reasonably well with the available experimental data and permit the extension of experimental observations into areas such as reaction zone thickness and the critical shear stress required for initiation.

Since the response of a crystalline explosive to any shock or impact is dependent upon the degree and nature of initiation achieved by the stimulus, it should be possible to extend these results beyond the narrow concerns of detonation and predict the response of an explosive to any arbitrary shock or impact. 


\section{ACKNOWLEDGEMENTS}

This work was supported by the Office of Naval Research and by the Naval Surface Warfare Center Independent Research Funds. The author wishes to thank Dr. Donald H. Liebenberg of the ONR and Mr. Raymond H. Riedl of NSWC for their support and encouragement. In particular, the author wishes to express his appreciation to Dr. E. R. Lemar of NSWC for his comments and insights and for kindly supplying and reducing the experimantal data and to Dr. R. H. Guirguis of NSWC.

Table (1) Comparison of typical experimental data for two explosives, Comp B and an AP/Al based material, with their predicted behavior.

Comp B

Charge radius $=25.43 \mathrm{~mm}$,

Measured from experiment, $\alpha=.01134 \mathrm{~mm}^{-1}, z_{0}=1.86 \mathrm{~mm}$

Reaction zone length calculated from Eq. $(10), \delta=.93 \mathrm{~mm}$

Predict $\mathrm{z}_{0}=2 \delta=1.86 \mathrm{~mm}$

AP/Al Explosive

Charge radius $=34.125 \mathrm{~mm}$

Measured from experiment, $\alpha=.018367 \mathrm{~mm}^{-1}, \mathrm{z}_{0}=5.48 \mathrm{~mm}$

Reaction zone length calculated from Eq. (10), $\delta=2.7 \mathrm{~mm}$

Predict $\mathrm{z}_{0}=2 \delta=5.4 \mathrm{~mm}$

AP/AI Explosive

Charge radius $=24.06 \mathrm{~mm}$

Measured from experiment, $\alpha=.0324 \mathrm{~mm}^{-1}, \mathrm{z}_{0}=4.88 \mathrm{~mm}$

Reaction zone length calculated from Eq. (10), $\delta=2.34 \mathrm{~mm}$

Predict $\mathrm{z}_{0}=2 \delta=4.68 \mathrm{~mm}$

Data was fit to equation (7) and both $\alpha$ and $z_{0}$ were determined (Courtesy of E.R. Lemar). Using $\alpha$ the reaction length $\delta$ was determined from equation $(10)$ and compared with the prediction $z_{0}=2 \delta$. It is not clear why the length of the reaction zone, $\delta$, differs by $.36 \mathrm{~mm}$ between the two AP/Al explosive entries above. It may be that the assumption that $\delta=$ constant independent of charge diameter is not totally correct. It could also be due to slight unintended compositional variations in the material or to experimental error.

\section{REFERENCES}

[1] Courant, R. and Friedrichs, K., Supersonic Flow and Shock Waves, (Interscience Publishers, Inc. New York, 1948).

[2] Zeldovich, Ya. B., Zh. Eksp. Teor. Fiz. 10, (1940) 542.

[3] Von Neumann, J., (1942) In Collected Works of John Von Neumann, Vol. 6, ed. A. J. Taub. (Macmillan, New York, 1963).

[4] Doering, W., Ann. Phys. 43 (1943) 421.

[5] Fickett, W. and Davis, W. C., In Detonation, (University of California Press, Berkeley, California, 1979).

[6] Lee, E. L. and Tarver, C. M., Phys. of Fluids 23, (1980) 2362.

[7] Johnson, J., Tang, P and Forest, C. A., J. Appl. Phys. 57, (1985) 4323.

[8] Bdzil, J. B. and Stewart, D. S., Phys. of Fluids A, 1 (7), (1989) 1261.

[9] Coffey, C. S., Phys. Rev. B 24, (1981) 6984.

[10] Coffey, C. S., Phys. Rev. B 32, (1984) 5335. 
[11] Coffey, C. S., J. Appl. Phys. 70 (8), (1991) 4248.

[12] Gilman, J. J., Aust. J. Phys. 13, (1960) 327.

[13] Gilman, J. J. and Johnston, W. G., in Solid State Physies, edited by F. Seitz and D. Turnbull (Academic, New York, 1962), Vol. 13, p. 147.

[14] Coffey, C. S., Submitted Phys. Rev. B. August 1994.

[15] Oeconomos, J.N. and Chaisse, F., To be published in the Proc. of the 10th Symp. on Detonation, (1993).

[16] Lemar, E. R., Private Communication, provided Figures 3 and 4.

[17] Forbes, J. W. and Lemar, E. R. Private Communication.

[18] Price, D. and Liddiard, T. P., NOLTR 66-87, Naval Ordnance Laboratory, White Oak, MD. (1966).

[19] Price, D.; Clairmont, A. R. and Erkman, J. O., NOLTR 74-40, Naval Ordnance Laboratory, White Oak, MD. (1974).

[20] Price, D., Lecture 9, NSWC MP 81-399, (1981).

[21] Spear, R. J. and Nanut, V. in Proc. of the 9th Symp. on Detonation, OCNR 113291-7, (1989) p. 98. 